\title{
On the Hopf Algebra Structure of the mod 2 Cohomology of a Finite $H$-Space
}

By

James LiN*

\section{$\S 1$. Introduction}

The action of the Steenrod algebra on the cohomology of a finite $H$-space has placed several restrictions on the possible spaces which can be finite $H$-spaces. It is now known, for example, that the loop space of a finite $H$-space has no homology torsion. We continue this study here by showing that in the mod 2 cohomology of a finite $H$-space, every generator of degree $4 k+1$ is in the image of $S q^{2 k}$.

This result has several consequences. For example, if an integer $n$ has dyadic expansion

$$
n=2^{s_{1}}+\cdots+2^{s_{l}}
$$

where $0 \leqq s_{\iota} \leqq s_{i+1}$ we say $n$ has length $l$. If $4 k+1$ has length $l$ and there is a generator of the $\bmod 2$ cohomology of a finite $H$-space in degree $4 k+1$, then there is a generator in degree $2^{l}-1$. Hence if a finite $H$-space is highly connected this result implies the non-existence of generators of small length.

Another implication is that there are no $4 k$ dimensional primitive indecomposables in the mod 2 cohomology of the loop space. Also, all generators of degree $8 k+5$ in the cohomology of a finite $H$-space are in the image of $S q^{2}$.

Throughout this paper it is assumed that all finite $H$-spaces used in this paper are simply connected and have associative mod 2 homology rings. Under this assumption it is easily shown that finite $H$-spaces that have no two torsion have primitively generated mod 2 cohomology rings. Hence their structure over the Steenrod algebra is restricted by several results of Thomas [4]. It is therefore more interesting to study finite $H$-spaces that have two torsion in their homology.

Under these circumstances it was shown that the two torsion is of order at most two and arises from odd degree mod 2 cohomology generators whose cup product is non trivial. In chapter 3 of this paper a structure theorem is given for the action of the Steenrod algebra on these generators. In particular if $y$ is

Communicated by N. Shimada, September 7, 1983.

* University of California, San Diego La Jolla, California 92093, USA.

** Partially supported by a National Science Foundation grant. 
a generator of the mod 2 cohomology of a finite $H$-space and the degree of $y$ has the form $2^{s+1}-1+2^{s+2} k$ where $s \geqq 0$ and $k>0$, and $y$ has cup product square non trivial, then $y$ is in the image of $S q^{2^{s+1_{k}}}$ modulo decomposables. For example, if $y$ is a generator in $H^{19}\left(X ; Z_{2}\right)$ where $X$ is a finite $H$-space and $y^{2}$ is non-zero, then $19=2^{2}-1+2^{4}$, hence $y=S q^{8} z$ modulo decomposables, where $z$ lies in $H^{11}\left(X ; Z_{2}\right)$. Applying the theorem again, $z=S q^{ \pm} w$ modulo decomposables where $w$ lies in $H^{7}\left(X ; \boldsymbol{Z}_{2}\right)$. So this theorem restricts the structure of the two torsion which appears in the mod 2 cohomology of a finite $H$-space.

In chapter four a general Hopf algebra structure theorem for the mod 2 cohomology of a finite $H$-space is presented. If $X$ is a finite $H$-space, $H^{*}\left(X ; Z_{2}\right)$ can be considered a universal object on a certain coalgebra which admits a squaring map. Let $R$ be the set of elements of $H^{*}\left(X ; Z_{2}\right)$ which have reduced coproduct in $\xi H^{*}\left(X ; \boldsymbol{Z}_{2}\right) \otimes H^{*}\left(X ; \boldsymbol{Z}_{2}\right)$. Note that this is a coalgebra over the Steenrod algebra, and the squaring map $\xi: H *\left(X ; \boldsymbol{Z}_{2}\right) \rightarrow H *\left(X ; \boldsymbol{Z}_{2}\right)$ in fact restricts to $R$. Such a coalgebra will be called a coalgebra with squares. Let $S(R)$ be the free commutative algebra on $R$ and let $I$ be the ideal in $S(R)$ generated by elements of the form $x^{2}-\xi x$. Then $S(R) / I$ is isomorphic to $H^{*}\left(X ; \boldsymbol{Z}_{2}\right)$ as Hopf algebras over the Steenrod algebra. Hence $H^{*}\left(X ; \boldsymbol{Z}_{2}\right)$ has a particularly simple form.

The author is grateful to Alex Zabrodsky and John C. Moore for several useful conversations. The author also thanks the Institute for Advanced Study in Jerusalem for their warm hospitality while work was in progress. Throughout the paper it will be assumed that the reader is familiar with the notation of [2].

\section{§2. $\boldsymbol{Q}^{4 k+1}=S \boldsymbol{q}^{2 k} \boldsymbol{Q}^{2 k+1}$}

In this chapter, we prove $Q^{1 k+1}=S q^{2 k} Q^{2 k+1}$ for $k \geqq 1$. The proof uses several techniques which appear in [2].

Definition. Let $v(s)=2^{s+1}-1$,

$$
\begin{aligned}
& a(s, k)=2^{s+2} k+2 v(s-1), \\
& b(s, k)=2^{s+2} k+2 v(s) .
\end{aligned}
$$

Let $I_{1}$ be the two sided ideal of $\mathcal{A}(2)$ generated by $S q^{2}, I_{2}$ the two sided ideal generated by $I_{1} \cdot I_{1}$. Let $J_{r}$ be the right ideal generated by $S q^{4}, \cdots, S q^{2^{r}}$ for $r \geqq 2$. Let $K_{r}$ be the right ideal generated by $J_{r}+I_{2}$.

The following theorems were proved in [2]:

Theorem 2.1 [2]. Let $s \geqq 1, k>1$, then

$\left(\mathrm{a}_{s}\right) \quad Q^{a(s, k)-1}=S q^{2^{s+1} k} Q^{b(s-1, k)-1}$.

(bs) $S q^{2^{s+1}} Q^{a(s, k)-1}=0$.

$\left(\mathbf{c}_{s}\right)$ If $t \in P_{b(s, k)-1}$, then $t$ is annihilated by $K_{s+1}$. Dually $Q^{b(s, k)-1} \cap \operatorname{im} K_{s+1}$ $=0$. 
(d $\mathrm{d}_{s} \quad Q^{2^{s+2}+2 v(s-1)-1}=S q^{2^{s+1}} Q^{2 v(s)-1}+S q^{2 v(s-1)} Q^{2^{s+2}-1}$.

(e) $S q^{4 l+2} Q^{1 k+1}=0$.

Theorem $2.2[2] . \quad Q^{2 v(\jmath)-1}=\bar{Q}_{\jmath_{-1}} Q^{2 v(\jmath-1)+1}$ where $\bar{Q}_{\jmath-1}$ is $S q^{0, \cdot 2}$, where 2 is in the $j^{\text {th }}$ place.

Definition. A doubleton $\beta \in \mathcal{A}(2)$ of degree $2^{r}$ is a sum of monomials of the form $b_{1} S q^{2^{i}} b_{2} S q^{2 i} b_{3}$ where $\operatorname{deg} b_{2} \equiv \operatorname{deg} b_{3} \equiv 0 \bmod 2^{i+1}$ and $i \leqq r$.

It is easy to see that $\bar{Q}_{\jmath_{-1}}=\left[S q^{2}, \bar{Q}_{\jmath-2}\right]+\delta$ where $\delta$ is a doubleton degree 1 .

We now consider several consequences of assuming

$$
Q^{2^{s+2}+2 v(s-1)-1}=S q^{2^{s+1}} Q^{2 v(s)-1} \quad \text { for } \quad s \geqq 1 .
$$

Theorem 2.3. If equation (2.1) holds, then Theorem 2.1 is true for $k=1$.

Proof. $\quad a(s, 1)-1=2^{s+2}+2 v(s-1)-1$ so $\left(a_{s}\right)$ is precisely equation (2.1). Now for $s=1$, equation (2.1) becomes

$$
Q^{9}=S q^{1} Q^{5}
$$

and $S q^{4} S q^{4}=S q^{7} S q^{1}+S q^{6} S q^{2}$. Since $S q^{2} Q^{1 k+1}=0$ and all doubletons of degree 1 annihilate $Q^{1 k+1}$ it follows that

$$
S q^{1} Q^{9}=S q^{1} S q^{1} Q^{5}=0 .
$$

We assume now by induction that

$$
S q^{2 t+1} Q^{2 t+2+2 v(t-1)-1}=0 \quad \text { for } t<s .
$$

Then

Consider

$$
\begin{aligned}
S q^{2^{s+1}} Q^{2^{s+2}+2 v(s-1)-1} & =S q^{2^{s+1}} S q^{2^{s+1}} Q^{2 v(s)-1} \text { by }(2.1) \\
& =\delta Q^{2 v(s)-1} \text { where } \delta \text { is a doubleton of } \\
& \text { degree } 2^{s} \text { by the Adem relations. }
\end{aligned}
$$

$$
b_{2} S q^{2 l} b_{3} Q^{2 v(s)-1} \subseteq Q^{a(i, k)-1} .
$$

By Theorem $2.1\left(\mathrm{~b}_{s}\right)$ and the inductive assumption (for $k=1$ ), it follows that

$$
S q^{2 i} b_{2} S q^{2 i} b_{3} Q^{2 v(s)-1}=0 \text {. }
$$

We conclude $\delta Q^{2 v(s)-1}=0$. Therefore, by induction we have shown $S q^{q^{s-1}} Q^{a(s, 1)-1}$ $=0$.

To prove $\left(\mathrm{c}_{s}\right)$ for $k=1$ note that $b(s, 1)=b(s-1,3)$ so

$$
Q^{b(s, 1)-1} \cap \operatorname{im} K_{s}=0 \quad \text { by Theorem } 2.1 \quad\left(c_{s-1}\right) .
$$

Note $b(s, 1)=2 v(s+1)$. Now if $Q^{b(s, 1)-1} \cap \operatorname{im} S q^{2 s+1} \neq 0$, then

$$
\begin{aligned}
Q^{2 v(s+1)-1} & =S q^{2^{s+1}} Q^{a(s, 1)-1} \\
& =S q^{2^{s+1}} S q^{2^{s+1}} Q^{2 v(s)-1}=0 \quad \text { by }\left(\mathrm{b}_{s}\right) .
\end{aligned}
$$

So we conclude 


$$
Q^{v(s 1)-1} \cap \operatorname{im} K_{s+1}=0
$$

This completes the proof.

Q.E.D.

Theorem 2.4. If equation (2.1) holds then for $k \geqq 1$

$$
Q^{4 k+1}=S q^{2 k} Q^{2 k+1} .
$$

Proof. Recall if $4 k+1$ is not of the form $2 v(j)-1$ then $4 k+1=a(s, l)-1$ for some $s \geqq 1, l \geqq 1$. By Theorems 2.1 and 2.3, $Q^{4 k+1}=\alpha Q^{2 v(\jmath)-1}$ where $\alpha \in \_-\{(2) \text {. }$ Now by Theorem 2.2

$$
\begin{aligned}
Q^{2 v(\jmath)-1} & =\bar{Q}_{\jmath-1} Q^{2 v(j-1)+1} \\
& =\left[S q^{2^{j}}, \bar{Q}_{\jmath-2}\right] Q^{2 v(\jmath-1)+1}
\end{aligned}
$$

since doubletons of degree one annihilate $Q^{2 v(j-1)+1}$. By Theorem 2.3, since $2 v(j)-1=b(j-1,1)-1, Q^{2 v(\jmath)-1} \cap \operatorname{im} K_{j}=0$. It follows that

$$
\begin{aligned}
Q^{2 v(\jmath)-1} & =\bar{Q}_{\jmath-2} S q^{2 \jmath} Q^{2 v(\jmath-1)+1} \\
& =\bar{Q}_{\jmath-3} S q^{2 j-1} S q^{2 j} Q^{2 v(\jmath-1)+1} \\
& =\cdots \\
& =S q^{2} S q^{4} \cdots S q^{2 j} Q^{2 v(\jmath-1)+1} \\
& =S q^{2 v(\jmath-1)} Q^{2 v(\jmath-1)+1} .
\end{aligned}
$$

It follows that for every $k$, there exists $\alpha \in \mathcal{A}(2), j$ an integer such that

$$
Q^{4 k+1}=\alpha S q^{2 v(j-1)} Q^{2 v(j-1)+1} \text {. }
$$

Now the Adem relations imply for $l, m$ positive integers,

$$
S q^{2 l} S q^{m} Q^{m+1}=S q^{m+l} S q^{l} Q^{m+1} .
$$

Applying this rule to $\alpha$, which is a polynomial in the even Steenrod squares, we conclude

$$
Q^{1 k+1}=S q^{2 k} Q^{2 k+1} \text {. Q.E.D. }
$$

The following factorizations follow by induction using the methods in $[2$, Prop. 2.7].

Proposition 2.5. (a) $S q^{10}=S q^{8} S q^{2}+\bar{Q}_{1} S q^{4}+Q_{0} Q_{1} S q^{6}+Q_{1} S q^{7}$

(b) For $s \geqq 2$

$$
+Q_{2} S q^{3}+S q^{1} S q^{2} S q^{1} S q^{6}
$$

$$
\begin{aligned}
S q^{2^{s+2}+2 v(s-1)}= & \sum_{u=-1}^{s-2} S q^{2^{s+2}+4 v(s-2)-1 v(u)} \bar{Q}_{u+1} \\
& +\bar{Q}_{s} S q^{2^{s+1}}+\sum_{u=0}^{s-1} Q_{u} Q_{u+1} S q^{2^{s+2}+2^{s+1-2} u+1-2} u+2 \\
& +Q_{s} S q^{2^{s+1}-1}+S q^{1} S q^{2} S q^{1} S q^{2^{s+2}+4 v(s-2)-2}
\end{aligned}
$$


(c) $S q^{4 k+2}=\sum_{j=-1} S q^{1 k-1 v(\jmath)} \bar{Q}_{\jmath+1}+\sum a_{\imath} \alpha_{\imath}$ where $\operatorname{deg} \alpha_{\imath} \equiv 3 \bmod$.our. $S q^{2^{2}+2^{i+1} l}=S q^{2^{i}} S q^{2^{i+1} l}+$ doubletons of degree $\leqq i-1$.

Now by [2, Thm. 8.7], we may choose representatives for $Q^{\left.2^{s+2}+2 v, s-1\right)-1}$ that have reduced coproduct in $\xi^{2} H^{*} \otimes R$, where

$$
R=\left\{x \in H^{*} \mid \bar{J} x \subseteq \xi H^{*} \otimes H^{*}\right\} .
$$

$R$ is an $\mathcal{A}(2)$ module.

Proposition 2.6. If $Q^{2^{s+2}-2 v(s-1)-1}$ is not contained in im $S q^{3^{3}+1}$ then there exist elements $x$ with $\bar{J} x \in \xi^{2} H^{*} \otimes R, \quad 0 \neq \bar{x} \in Q^{2^{s+2}+2 v(s-1)-1}, \quad S q^{1} x=0$, and $\bar{x} \oplus$ $\operatorname{im} S q^{2 s+1}$.

Proof. By the above remark there is an $x^{\prime}$ with $\vec{x}^{\prime} \notin \mathrm{im} S q^{2^{3+1}}, \bar{J} x^{\prime} \in \bar{\zeta}^{2} H * \otimes R$, and $0 \neq \bar{x}^{\prime} \in Q^{2^{s+2}+2 v(s-1)-1}$. Now if $S q^{1} x^{\prime} \neq 0$ then

$$
\left\{S q^{1} x^{\prime}\right\} \in P\left(H^{*} / / \xi^{2} H^{*}\right)
$$

is decomposable since $Q^{\text {even }}=0$ by $\left[2\right.$, Cor. 7.6]. Hence $\left\{S q^{1} x^{\prime}\right\}=\left\{u^{\prime}\right\}^{2}$ where we may assume $w \in R$. If $u=S q^{1} x^{\prime}-w^{2}$ then $u \in I\left(\xi^{2} H^{*}\right) H^{*}$ and $J u \in \xi^{2} H^{*} \otimes H^{*}$ Applying the Milnor Moore isomorphism

$$
H^{*} \longrightarrow H^{*} \otimes H^{*} \longrightarrow H^{*} / / \xi^{2} H^{*} \otimes \xi^{2} H^{*}
$$

implies $u \in \xi^{2} H^{*}$. But deg $u \equiv 2 \bmod 4$ and $\xi^{2} H^{*}$ is concentrated in degrees divisible by 4. Therefore $u=0$ and

$$
\begin{aligned}
S q^{1} x^{\prime} & =w^{2} \\
& =S q^{1} S q^{2^{s+1}+2 v(s-2)} w .
\end{aligned}
$$

Hence $x=x^{\prime}-S q^{2^{s+1}+2 v(s-2)} u$ has $S q^{1} x=0$. By Theorem 2.1(c) and Proposition 2.5(c) we get

$$
S q^{2^{s+1}+2 v(s-2)} w=\sum S q^{2^{s+1}+1 v(s-3)-1 v(\jmath)} \bar{Q}_{\jmath-1} u .
$$

Now if $j \neq s-3$, a simple argument shows that

$$
\operatorname{im} S q^{s^{s+1}+1 v(s-3)-1 v(\jmath)} \subseteq \operatorname{im}\left(S q^{1}, S q^{2}, \cdots, S q^{2^{s}}\right) .
$$

By Theorem 2.1(c)

$$
S q^{2^{s+1}+2 v(s-2)} u=S q^{2^{s+1}} \bar{Q}_{s-2} u \text {. }
$$

Hence $x=x^{\prime}-S q^{2^{s+1}} \bar{Q}_{s-3} u$. It follows that $\bar{x} \in \mathrm{im} S q^{2^{s+1}}$. This completes the proof.

Q.E.D.

We now prove $Q^{9}=S q^{1} Q^{5}$. This is equation (2.1) for $s=1$. This simple example will illustrate the methods used. Later we will prove equation (2.1) for arbitrary $s$.

By Proposition 2.5(a) 


$$
\begin{aligned}
S q^{10}= & S q^{8} S q^{2}+\bar{Q}_{1} S q^{4}+Q_{0} Q_{1} S q^{6}+Q_{1} S q^{7}+Q_{2} S q^{3} \\
& +S q^{1} S q^{2} S q^{1} S q^{6} \\
= & \left(S q^{8}+Q_{2} S q^{1}\right) S q^{2}+\left(Q_{0} Q_{1}+Q_{1} S q^{1}+S q^{1} S q^{2} S q^{1}\right) S q^{6}+\bar{Q}_{1} S q^{4} .
\end{aligned}
$$

By Theorem 2.1(e), since $S q^{\top}=S q^{1} S q^{6}, S q^{3}=S q^{1} S q^{2}$,

$$
0=S q^{2} Q^{9}=S q^{6} Q^{9}=S q^{7} Q^{9}=S q^{3} Q^{9} .
$$

By Theorem 2.2,

$$
S q^{4} Q^{9} \subseteq \bar{Q}_{1} Q^{\top} \text { and } \bar{Q}_{1} \bar{Q}_{1}=0 \text {. }
$$

Our situation is that we can write $S q^{10}=\bar{Q}_{1} S q^{4}+\sum a_{\imath} b_{i}$ where if $\bar{x} \in Q^{9}$ with representative $x \in R$, then $b_{2} x=0$ and $S q^{4} x=\bar{Q}_{1} y$.

Both $x$ and $y$ may be chosen to be primitive. To see this, note that if $x \subseteq$ $R$ then $\bar{J} x \in \xi^{2} H^{*} \otimes R$. Now because $H^{*}\left(X ; Z_{2}\right)$ is trivial in degrees 1 and 2 , $\xi^{2} H^{*}$ is 11 connected. Hence $x$ is primitive. Similarly, $y$ may be chosen so that $\bar{J} y \in \xi H^{*} \otimes R$, and $\hat{\xi} H^{*}$ is 5 connected, so $y$ is primitive.

We now write down the universal example which describes the above relations. Let

$$
\begin{gathered}
K=K(\boldsymbol{Z}, 9,7) \\
K_{0}=K\left(\boldsymbol{Z}_{2}, 11,13,15\right) \\
w: K \longrightarrow K_{0} \\
w^{*}\left(i_{11}\right)=S q^{2} i_{9} \\
w^{*}\left(i_{13}\right)=S q^{4} i_{9}-\bar{Q}_{1} i_{\tau} \\
u^{*}\left(i_{15}\right)=S q^{6} i_{9} .
\end{gathered}
$$

Then

$$
z=\left(S q^{8}+Q_{2} S q^{1}\right) i_{11}+\left(Q_{0} Q_{1}+Q_{1} S q^{1}+S q^{1} S q^{2} S q^{1}\right) i_{15}+\bar{Q}_{1} i_{13} \in \operatorname{ker} w^{*} .
$$

In fact

$$
w^{*}(z)=S q^{10} i_{9}=0 .
$$

Let $E$ be the fibre of $w$. It follows that there is an element $v \in H^{*}\left(E ; \boldsymbol{Z}_{2}\right)$ with $J_{\imath}=u \otimes u$ where $u=p^{*}\left(i_{9}\right)$

$$
\begin{aligned}
j *(\imath)=\left(S q^{8}+Q_{2} S q^{1}\right) \sigma^{*}\left(i_{11}\right) & +\left(Q_{0} Q_{1}+Q_{1} S q^{1}+S q^{1} S q^{2} S q^{1}\right) \sigma^{*}\left(i_{15}\right) \\
& +\bar{Q}_{1} \sigma^{*}\left(i_{13}\right) . \\
& \Omega K_{0} \\
& \downarrow j \\
& E \\
& \downarrow p \downarrow \\
& \\
& \stackrel{\downarrow}{\longrightarrow} \longrightarrow K_{0} .
\end{aligned}
$$


The proof of this follows from [2, Prop. 3.1].

Now suppose $Q^{9}$ is not contained in the image of $S q^{4}$. Then there exists an $x \in R^{9}$, with $x \notin \operatorname{im} S q^{4}, x$ primitive, and a $y$ primitive such that $S q^{4} x=\bar{Q}_{1} y$. Hence there is a commutative diagram

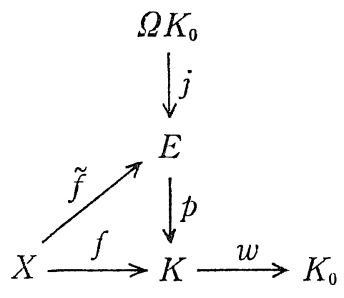

where $f^{*}\left(i_{9}\right)=x, f *\left(i_{7}\right)=y$. It follows that the $H$-deviation of $\tilde{f}$ factors through $2 K_{0}$ so we have $D_{\tilde{f}}=j \hat{D}$ and

$$
\begin{aligned}
\bar{J} \tilde{f}^{*}(v)= & \left(\tilde{f} * \otimes \tilde{f}^{*}\right)(\bar{J} v)+D_{\tilde{f}}^{*}(v) \\
= & x \otimes x+\hat{D}^{*} j^{*}(v) \\
\in & x \otimes x+\operatorname{im}\left(S q^{8}+Q_{2} S q^{1}\right) \\
& +\operatorname{im}\left(Q_{0} Q_{1}+Q_{1} S q^{1}+S q^{1} S q^{2} S q^{1}\right) \\
& +\operatorname{im} \bar{Q}_{1} .
\end{aligned}
$$

Now if $x \in \operatorname{im~} S q^{4}$, there exists a primitive $t \in P_{9}$ with $\langle t, x\rangle \neq 0$ and $t S q^{1}=0$. By Browder, if $\operatorname{deg} \alpha$ odd, $\alpha \in \mathcal{A}(2), t \alpha=0$ [1]. It follows that

$$
\begin{aligned}
\left\langle t^{2}, \tilde{f} *(v)\right\rangle= & \left\langle t \otimes t, \tilde{J} \tilde{f}^{*}(v)\right\rangle \\
= & \langle t \otimes t, x \otimes x\rangle+\left\langle t \otimes t, \operatorname{im}\left(S q^{8}+Q_{2} S q^{1}\right)\right\rangle \\
& -\left\langle t \otimes t, \operatorname{im}\left(Q_{0} Q_{1}+Q_{1} S q^{1}+S q^{1} S q^{2} S q^{1}\right)\right\rangle \\
& +\left\langle t \otimes t, \operatorname{im} \bar{Q}_{1}\right\rangle \\
= & \langle t, x\rangle^{2}+\left\langle t \otimes t, \operatorname{im} \bar{Q}_{1}\right\rangle .
\end{aligned}
$$

Now $\bar{Q}_{1}=\left[S q^{1}, S q^{2}\right]+S q^{1}$ doubletons

So $t \bar{Q}_{1}=0$ implies

$$
\bar{J} \bar{Q}_{1}=Q_{1} \otimes Q_{1} .
$$

$$
\left\langle t^{2}, \tilde{f} *(v)\right\rangle=\langle t, x\rangle^{2} \neq 0 \text {. }
$$

This is a contradiction because $t^{2}=0$ if $t \in P_{\text {odd }}$ by Browder [1].

We conclude that our original assumption that $Q^{9}$ is not contained in im $S q^{4}$ must be false. Therefore we have proven

Theorem 2.7. $Q^{9}=S q^{4} Q^{5}$.

To prove that $Q^{2^{s+2}+2 v(s-1)-1}=S q^{2^{s+1}} Q^{2 v(s)-1}$ is analogous to the above proof, 
we must construct the universal example, verify that primary operations vanish on our element and then examine the properties of the $H$-deviation. The only difference is that in general elements in the domain of the operation are not necessarily primitive. We therefore use the methods of Cartan formulae described in [5, Sec. 4]. We assume the reader is familiar with this and will use these results freely.

Now consider the factorization from Proposition 2.5(b).

$$
\begin{aligned}
S q^{2^{s+2}+q v(s-1)}= & \sum_{u=-1}^{s-2} S q^{s^{s+2}+1 v(s-2)-4 v(u)} \bar{Q}_{u+1}+\bar{Q}_{s} S q^{2^{s+1}} \\
& +\sum_{u=0}^{s-1} Q_{u} Q_{u+1} S q^{2^{s+2+2} s+1_{-2} u_{+1-2} u+2} \\
& +Q_{s} S q^{2^{s+1-1}}+S q^{1} S q^{2} S q^{1} S q^{2^{s+2}+4 v(s-2)-2} .
\end{aligned}
$$

Henceforth we assume $Q^{2^{s+2_{T}} v(s-1)-1}$ is not contained in $\mathrm{im} S q^{2^{s+1}}$. Our goal will be to produce a contradiction. We will find a $t \in P_{2 s+2+2 v(s-1)-1}$ with $t^{2} \neq 0$. This will contradict the result of Browder.

By Proposition 2.6 there is an $x$ with $0 \neq \bar{x} \equiv Q^{2^{s+2}+2 v(s-1)-1}$ and $S q^{1} x=0$ and $\bar{x} \Subset \mathrm{im} \mathrm{Sq^{2 }}{ }^{s+1}$. It follows that there is a primitive $t$ with

Further, $\bar{\Delta} x \in \xi^{2} H^{*} \otimes R$.

$$
\langle t, x\rangle \neq 0 \text { and } t S q^{2^{s+1}}=0 \text {. }
$$

By Theorem 2.1(e) we have

(a) $\bar{Q}_{u+1} x=0$ for all $u$.

(b) $S q^{2^{s+1}-1} x=S q^{1} S q^{2 s+1-2} x=0$.

(c) $S q^{2^{s+2}+4 v(s-2)-2} x=0$.

By Theorem 2.2, there is a $y \in R$ with

(d) $S q^{2^{s+1}} x=\bar{Q}_{s} y$. (Theorem 2.2 states that this is true modulo decomposables. A simple argument using the Milnor Moore isomorphism implies strict equality.)

Proposition 2.8. $Q_{u+1} S q^{2^{s+2}+2^{s+1}-2^{u+1}-2^{u+2}} x=0$ for all $u$ such that $0 \leqq u \leqq s-1$.

Proof. The case $u=0$ follows from Theorem 2.1(e). Recall that $Q_{0}=S q^{1}$, $Q_{1}=\left[S q^{2}, S q^{1}\right]$ and $Q_{i}=\left[S q^{2^{i-1}}, Q_{\imath-1}\right]$. Hence $Q_{u+1}$ is a sum of monomials with $S q^{1}$ and $S q^{2}$ in them. If $b_{1} S q^{1} b_{2} S q^{2} b_{3}$ is such a monomial, then degree $b_{3}$ must

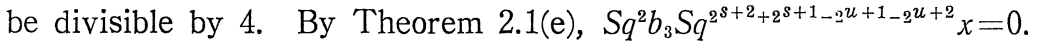

Now consider a monomial of the form $c_{1} S q^{2} c_{2} S q^{1} c_{3}$. If this is a summand of $Q_{u+1}$ then degree $c_{3}$ is divisible by 4 . The Adem relation

$$
S q^{1} S q^{4 l}=S q^{4 l} S q^{1}+S q^{2} S q^{1} S q^{4 l-2}
$$

and Theorem 2.1(e) imply for $u>0$, 


$$
\begin{aligned}
& c_{1} S q^{2} c_{2} S q^{1} c_{3} S q^{2^{s+2}+2^{s+1}-2^{u+1_{2}}{ }^{u+2} x} x \\
& \quad=c_{1} S q^{2} c_{2} c_{3} S q^{2^{s+2}+2^{s+1-2} u+1_{2} u+2} S q^{1} x \\
& =0 \text { because } S q^{1} x=0 .
\end{aligned}
$$

The theorem follows from the above arguments.

Q.E.D.

We now construct the universal example which describes the relations (a), (b), (c), (d) and Proposition 2.8 .

Let $K=K\left(\boldsymbol{Z}_{2}, 2^{s+2}+2 v(s-1)-1,2^{s+2}-1\right)$. Let $i$ be the fundamental class in degree $2^{s+2}+2 v(s-1)-1, \eta$ be the fundamental class of degree $2^{s+2}-1$

$$
\begin{aligned}
& K_{0}=\sum_{u=-1}^{s-2} K\left(\boldsymbol{Z}_{2}, 2^{s+2}+2 v(s-1)+2 v(u+1)-1\right) \\
& \times K\left(\mathbb{Z}_{2}, 2 v(s+1)-1\right) \\
& \times \sum_{u=0}^{s-1} K\left(\boldsymbol{Z}_{2}, 2^{s+3}+2 v(s)-2^{u+1}-2\right) \\
& \times K\left(\boldsymbol{Z}_{2}, 2 v(s+1)-2,2^{s+3}+8 v(s-2)-1\right) 。 \\
& w: K \longrightarrow K_{0} \\
& w^{*}\left(i_{2 s+2+2 v(s-1)+2 v(u+1)-1}\right)=\bar{Q}_{u+1} i \\
& w^{*}\left(i_{2 v(s+1)-1}\right)=S q^{2^{s+1}} i-\bar{Q}_{s} \eta \\
& w^{*}\left(i_{2} s+3+2 v(s)-2 u+1-2\right)=Q_{u+1} S q^{2 s+2+2 s+1-2 u+1-2 u+2} i \\
& w^{*}\left(i_{2 v(s+1)-2}\right)=S q^{2 s+1-1} i \\
& w *\left(i_{2 s+3+8 v(s-2)-1}\right)=S q^{2^{s+2}+4 v(s-2)-2} i .
\end{aligned}
$$

Let $E$ be the fibre of $w$

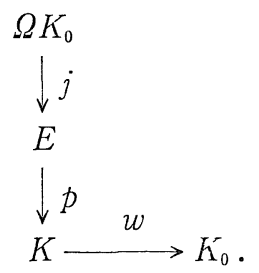

The argument in $[2, \operatorname{Sec} .3]$ implies there is a $v \in H^{*}\left(E ; \mathbb{Z}_{2}\right)$ with

$$
\bar{J} v=u \otimes u, u=p^{*}(i)
$$

and

$$
\begin{aligned}
& j *(v)=\sum_{u=-1}^{s-2} S q^{2^{s+2}+t v(s-2)-t v(u)} \sigma^{*}\left(i_{2 s+2+2 v(s-1)+2 v(u+1)-1}\right) \\
& +\bar{Q}_{s} \sigma^{*}\left(i_{2 v(s+1)-1}\right)
\end{aligned}
$$




$$
\begin{aligned}
& +\sum_{u=0}^{s-1} Q_{u} \sigma^{*}\left(i_{2 s+3+2 v(s)-2^{u+1-2}}\right) \\
& +Q_{s} \sigma^{*}\left(i_{2 v(s+1)-2}\right) \\
& \left.+S q^{1} S q^{2} S q^{1} \sigma^{*\left(i_{2} s+3+8 v(s-2)-1\right.}\right) .
\end{aligned}
$$

There is a commutative diagram

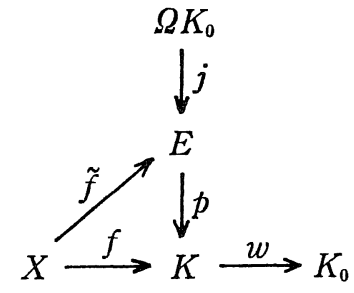

and $f^{*}(i)=x, f^{*}(\eta)=y, \bar{J} \tilde{f}^{*}(v)=x \otimes x+D_{f}^{*}(v)$.

In $[2,5$, Sec. 4$]$ the theory of Cartan maps describes how to compute $D_{\widetilde{f}}^{*}(v)$. Note there is a commutative diagram

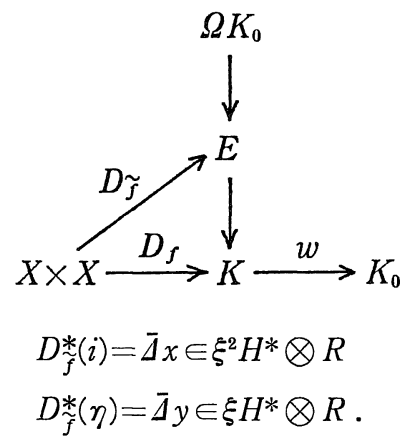

Hence there exist generalized Eilenberg MacLane spaces $A_{1}$ concentrated in even degrees and $A_{2}$ concentrated in odd degrees such that $D_{f}$ factors

$$
X \times X \stackrel{f_{1} \times f_{2}}{\longrightarrow} A_{1} \times A_{2} \stackrel{m}{\longrightarrow} K
$$

and $m$ is a Cartan map. That is, there exist $K_{1}, K_{2}$ and maps $w_{i}, n_{i}$ such that there is a commutative square

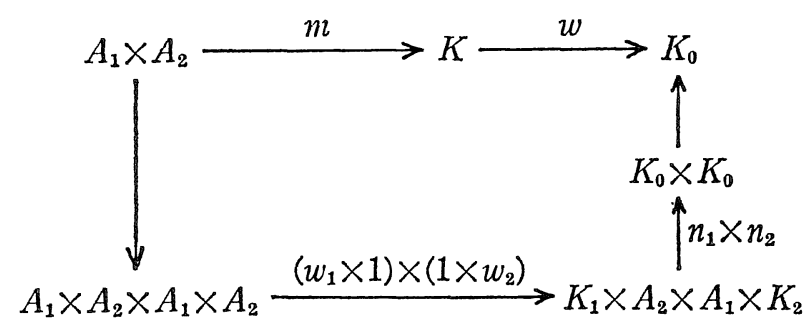


By [2, Theorem 4.1] if $E_{\imath}$ is the fibre of $w_{\imath}$, there is a commutative diagram

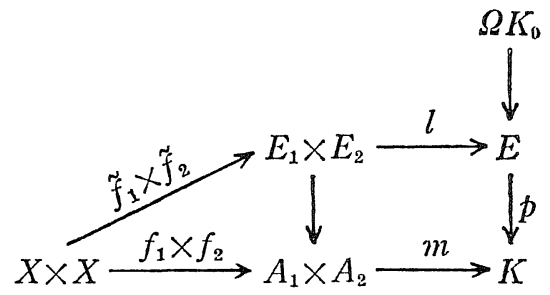

Hence $D_{\tilde{f}}$ and $l\left(\tilde{f}_{1} \times \tilde{f}_{2}\right)$ both lift $D_{f}$ so

$$
D_{\tilde{f}}^{*}(v)=\left(\tilde{f}_{1} \times \tilde{f}_{2}\right) * l *(v)+\hat{D}^{*} j^{*}(v)
$$

for some $\hat{D}: X \times X \rightarrow \Omega K_{0}$.

By Theorem 4.1 of $[2]$

$$
\left(\tilde{f}_{1}^{*} \otimes \tilde{f}_{2}^{*}\right) l^{*}(v) \subseteq \operatorname{im} f_{1}^{*} \otimes H^{*}+H^{*} \otimes \operatorname{im} f_{2}^{*} .
$$

Now $\operatorname{im} f_{1}^{*} \subseteq \xi H^{*}$, hence this part has no summand in an odd degree. We therefore concentrate on the contribution of $\left(\tilde{f}_{1}^{*} \otimes \tilde{f}_{2}^{*}\right) l^{*}(v)$ in $H^{*} \otimes i \mathrm{im} f_{2}^{*}$ in bidegrees $\left(2^{s+2}+2 v(s-1)-1,2^{s+2}+2 v(s-1)-1\right)$.

Let $F_{m} R=\left\{z \in R \mid \bar{J}^{m} z=0\right\}$. Let $C(m)$ be the $\mathcal{A}(2)$ subHopf algebra of $H^{*}$ generated by $F_{m} R$. Then $H^{*}$ is filtered

$$
\boldsymbol{Z}_{2} \subseteq C(1) \subseteq C(2) \subseteq \cdots \subseteq H^{*} .
$$

Assume $x \in C(m), x \in C(m-1)$. By coassociativity $J x \in C(m-1) \otimes C(m-1)$. We can clearly choose $t$ so that $\langle t, C(m-1)\rangle=0$.

Theorem 2.9. $\left\langle t \otimes t,\left(\tilde{f}_{1}^{*} \otimes \tilde{f}_{2}^{*}\right) l^{*}(v)\right\rangle=0$.

Proof. We follow closely [2, Sec. 4]. Given a fundamental class $i$

$$
m^{*} w^{*}(i)=\sum_{j} \hat{a}_{\jmath} \otimes a_{\jmath}+\sum_{k} b_{k} \otimes \hat{b}_{k}
$$

where $\hat{a}_{\jmath} \in \operatorname{ker} f_{1}^{*}, \hat{b}_{k} \in \operatorname{ker} f_{2}^{*}, f_{1}^{*}\left(b_{k}\right) \in \xi H^{*}, f_{2}^{*}\left(a_{\jmath}\right) \in R$.

Define

$$
\begin{array}{ll}
K_{1}=\Pi K\left(\boldsymbol{Z}_{2}, \operatorname{deg} \hat{a}_{\jmath}\right) & K_{2}=\Pi K\left(\boldsymbol{Z}_{2}, \operatorname{deg} \hat{b}_{k}\right) \\
w_{1}: A_{1} \longrightarrow K_{1}^{*} & w_{1}^{*}\left(i_{\operatorname{deg} \hat{a}_{\jmath}}\right)=\hat{a}_{3} \\
w_{2}: A_{2} \longrightarrow K_{2} & w_{2}^{*}\left(i_{\operatorname{deg} \hat{b}_{k}}\right)=\hat{b}_{k} \\
n_{1}: K_{1} \times A_{2} \longrightarrow K_{0} & n_{1}^{*}(i)=\sum i_{\operatorname{deg}} \hat{a}_{j} \otimes a_{J} \\
n_{2}: A_{1} \times K_{2} \longrightarrow K_{11} & n_{3}^{*}(i)=\sum b_{k} \otimes i_{\operatorname{deg} \hat{b}_{k}} .
\end{array}
$$

$n_{1}, n_{2}$ induce maps 


$$
\begin{aligned}
& k_{1}: \Omega K_{1} \wedge E_{2} \longrightarrow \Omega K_{0} \\
& k_{2}: E_{1} \wedge \Omega K_{2} \longrightarrow \Omega K_{0} \\
& k_{1}^{*}\left(\sigma^{*}(i)\right)=\sum_{\jmath} \sigma^{*}\left(i_{\operatorname{deg}} \hat{a}_{j}\right) \otimes p_{2}^{*}\left(a_{\jmath}\right) \\
& k_{2}^{*}\left(\sigma^{*}(i)\right)=\sum_{k} p_{1}^{*}\left(b_{k}\right) \otimes \sigma^{*}\left(i_{\operatorname{deg} \hat{b}_{k}}\right) .
\end{aligned}
$$

From this we can compute $k_{1}^{*} j^{*}(v), k_{2}^{*} j^{*}(v)$

$$
\begin{aligned}
& k_{1}^{*} j^{*}(v)=\sum \kappa_{j}^{\prime} \otimes p_{2}^{*}\left(a_{j}^{\prime \prime}\right) \\
& k_{2}^{*} j^{*}(v)=\sum p_{1}^{*}\left(a_{i}^{\prime}\right) \otimes \kappa_{\imath}^{\prime \prime}
\end{aligned}
$$

where $j^{*}\left(v_{\jmath}^{\prime}\right)=\kappa_{\jmath}^{\prime}, j^{*}\left(v_{2}^{\prime \prime}\right)=\kappa_{i}^{\prime \prime}$. From this

$$
\begin{array}{r}
\left(\tilde{f}_{1}^{*} \otimes \tilde{f}_{2}^{*}\right) l^{*}(v)=\sum \tilde{f}_{1}^{*}\left(v_{j}^{\prime}\right) \otimes f_{2}^{*}\left(a_{j}^{\prime \prime}\right)+\sum f_{1}^{*}\left(a_{i}^{\prime}\right) \otimes f_{2}^{*}\left(v_{\imath}^{\prime \prime}\right) \\
\text { modulo image }\left(f_{1}^{*} \otimes f_{2}^{*}\right) .
\end{array}
$$

Clearly the second summation belongs to $\xi H^{*} \otimes H^{*}$. These operations annihilate $t \otimes t$ for degree reasons.

Now consider the first summand. Let $S_{1}$ be the $A(2)$ span of terms in the second bidegree of $m *(i)$ and let $S_{2}$ be the $\mathcal{A}(2)$ span of terms in the second bidegree of $m^{*}(\eta)$. By construction $a_{j}^{\prime \prime} \in S_{1}+S_{2}$.

Case 1. If $a_{j}^{\prime \prime} \in S_{1}$ then $f_{2}^{*}\left(a_{j}^{\prime \prime}\right) \in C(m-1)$ and hence a term of this form annihilates $t \otimes t$.

Case 2. If $a_{\jmath}^{\prime \prime} \in S_{2}$ and $\left\langle t, f_{2}^{*}\left(a_{j}^{\prime \prime}\right)\right\rangle \neq 0$ we must have degree $f_{2}^{*}\left(a_{j}^{\prime \prime}\right)=2^{s+2}+$ $2 v(s-1)-1$.

Tracing backwards through the construction, we must have $j^{*}\left(v_{\jmath}^{\prime}\right) \otimes p_{2}^{*}\left(a_{\jmath}^{\prime \prime}\right)$ appearing in

$$
\bar{Q}_{s} k_{1}^{*} \sigma^{*}\left(i_{2 v(s+1)-1}\right) .
$$

Since $\bar{J} \bar{Q}_{s}=Q_{s} \otimes Q_{s}$ and $t Q_{s}=0$ we can reduce to the situation where the secondary operations comes from

$$
j *\left(v_{\jmath}^{\prime}\right)=\bar{Q}_{s} \sigma^{*}\left(i_{\operatorname{deg}} \hat{a}_{\jmath}\right) .
$$

But then $f_{2}^{*}\left(a_{j}^{\prime \prime}\right)$ must be an element in the second bidegree of a summand of $J^{\prime}$. Because degree $y$ is less than degree $x$, degree $f_{2}^{*}\left(a_{\jmath}^{\prime \prime}\right)<$ degree $x$. Hence

$$
\left\langle t, f_{2}^{*}\left(a_{\jmath}^{\prime \prime}\right)\right\rangle=0 \text {. }
$$

Finally, $\operatorname{im}^{*} f_{1}^{*} \otimes f_{2}^{*} \subseteq \xi H^{*} \otimes R$ so this also annihilates $t \otimes t$.

We conclude $\left\langle t \otimes t,\left(\tilde{f}_{1}^{*} \otimes \tilde{f}_{2}^{*}\right) l^{*}(v)\right\rangle=0$.

It follows that

$$
\begin{aligned}
\left\langle t^{2}, \tilde{f}^{*}(v)\right\rangle & =\left\langle t \otimes t, \bar{J} \tilde{f}^{*}(v)\right\rangle \\
& =\left\langle t \otimes t, x \otimes x+\left(\tilde{f}_{1}^{*} \otimes \tilde{f}_{2}^{*}\right) l^{*}(v)+\hat{D}_{j}^{*}(v)\right\rangle
\end{aligned}
$$




$$
\begin{aligned}
& =i t, x\rangle^{2}+\left\langle t \otimes t, \hat{D} j^{*}(v)\right\rangle \text { by Theorem } 2.9 \\
& =\langle t, x\rangle^{2}+\left\langle t \otimes t, \sum_{u=-1}^{s-2} \operatorname{im} S q^{2 s+2+4 v(s-2)-4 v(u)}\right\rangle \\
& \left.\quad+i t \otimes t, \operatorname{im} \bar{Q}_{s}\right\rangle+\left\langle t \otimes t, \sum_{u=0}^{s} \operatorname{im} Q_{u}\right\rangle \\
& \quad+\left\langle t \otimes t, \operatorname{im} S q^{1} S q^{2} S q^{1}\right\rangle .
\end{aligned}
$$

Since $t \alpha=0$ for $\operatorname{deg} \alpha$ odd, only the first two terms besides $\langle t, x\rangle^{2}$ can possibly be nonzero.

Step 1. 〈t $\left.\otimes t, \Sigma \mathrm{im} S q^{2 s+2_{+}+v(s-2)-4 v(u)}\right\rangle$. We must consider terms of the form

$$
t S q^{2 s+1+2 v(s-2)-2 v(u)+l} \otimes t S q^{2^{s+1}+2 v(s-2)-2 v(u)-l}
$$

where $-2 v(u) \leqq l \leqq 2 v(u)$.

By Theorem 2.1(c) and the assumption $t S q^{2^{s+1}}=0, t$ is annihilated by $K_{s+1}$. By checking degrees, Theorem 2.1(c) implies $t$ is annihilated by doubletons of degree $\leqq s . \quad$ Now if degree $2^{s+1}+2 v(s-2)-2 v(u)+l \equiv 2 \bmod 4$ Proposition 2.5(c) implies

$\operatorname{im} S q^{2^{s+1}+2 v(s-2)-2 v(u)+l} \subseteq \operatorname{im} S q^{2^{i}}+\mathrm{im} S q^{2^{s+1}}+$ doubletons of degree $\leqq S$

$$
\text { for some } i \leqq s \text {. }
$$

If degree $2^{s+1}+2 v(s-2)-2 v(u)+l \equiv 0 \bmod 4$, Proposition 2.5(c) implies

$$
\text { im } S q^{2^{s+1}+2 v(s-2)-2 v(u)+l} \subseteq \operatorname{im~} S q^{2^{i}}+\text { doubletons of degree } \leqq i-1
$$

for some $i \leqq s$.

In all cases

$$
\left\langle t \otimes t, \operatorname{im} S q^{2 s+2+1 v(s-2)-1 v(u)}\right\rangle=0 .
$$

Step 2. $\left\langle t \otimes t, \operatorname{im} \bar{Q}_{s}\right\rangle$.

$$
\bar{Q}_{s}=\left[S q^{2 s+1}, \bar{Q}_{s-1}\right],(t \otimes t) \bar{Q}_{s}=t \bar{Q}_{s} \otimes t+t \otimes t \bar{Q}_{s} .
$$

If $t \bar{Q}_{s} \neq 0$ then $\bar{Q}_{s} Q^{2^{s+1}-1}=S q^{q^{s+1}} \bar{Q}_{s-1} Q^{2^{s+1}-1}$. Hence $t \bar{Q}_{s} \neq 0$ implies $t S q^{2^{s+1}} \neq 0$. But by assumption $t S q^{2^{s+1}}=0$. Therefore $\left\langle t \otimes t\right.$, im $\left.\bar{Q}_{s}\right\rangle=0$.

We conclude $\left\langle t^{2}, \tilde{f}^{*}(v)\right\rangle=\langle t, x\rangle^{2}$ which implies $t^{2} \neq 0$. This is the desired contradiction.

We have proved the following theorem:

Theorem 2.10. $Q^{2^{s+2}-2 v(s-1)-1}=S q^{2 s+1} Q^{2 v(s)-1}$ for $s \geqq 1$. Hence by' Theorem 2.4, $Q^{1 k+1}=S q^{2 k} Q^{2 k+1}$.

\section{§3. $\xi H^{*}\left(X ; Z_{2}\right)$}

In this chapter we prove a theorem about algebra generators of $H^{*}\left(X ; Z_{3}\right)$ 
whose cup product square is nontrivial. The results derive from techniques developed by Zabrodsky [6]. Because $H_{*}(\Omega X ; \boldsymbol{Z})$ is two torsion free it was shown that

$$
\text { Tor }_{H^{*}\left(X ; \boldsymbol{Z}_{2}\right)}\left(\boldsymbol{Z}_{2}, \boldsymbol{Z}_{2}\right) \text { is isomorphic to } H^{*}\left(\Omega X ; \boldsymbol{Z}_{2}\right)
$$

as a coalgebra [2].

If $H^{*}\left(X ; \boldsymbol{Z}_{2}\right)=\bigotimes_{i} \wedge\left(x_{i}\right) \bigotimes_{j} \boldsymbol{Z}_{2}\left[y_{j}\right] /\left(y_{j}\right)^{2_{j}}$ then there is an exterior coalgebra $\wedge\left(\sigma^{*}\left(y_{j}\right)\right) \subseteq H^{*}\left(\Omega X ; \boldsymbol{Z}_{2}\right)$. In $H_{*}\left(\Omega X, \boldsymbol{Z}_{2}\right)$ this corresponds to a subHopf algebra which is the kernel of $\xi: H_{*}\left(\Omega X ; \boldsymbol{Z}_{2}\right) \rightarrow H_{*}\left(\Omega X ; \boldsymbol{Z}_{2}\right)$.

Since

$$
\operatorname{Tor}_{H^{*}\left(X ; Z_{2}\right)}\left(\boldsymbol{Z}_{2}, \boldsymbol{Z}_{2}\right)=\Gamma\left(\sigma^{*}\left(x_{i}\right)\right) \otimes \wedge\left(\sigma^{*}\left(y_{j}\right)\right) \otimes \Gamma\left(\varphi_{2} f_{\jmath}\left(y_{\jmath}\right)\right)
$$

it follows that all generators of kernel $\xi$ map monomorphically to $H_{*}\left(X ; \boldsymbol{Z}_{2}\right)$ This implies the following theorem:

Theorem 3.1. Let $y \in H^{*}\left(X ; \boldsymbol{Z}_{2}\right)$ have degree $v(s)+2^{s+2} k, s \geqq 0, k>0$ and suppose $y^{2} \neq 0$ and $\bar{\Delta} y \in \xi H^{*}\left(X ; \boldsymbol{Z}_{2}\right) \otimes H^{*}\left(X ; \boldsymbol{Z}_{2}\right)$. Then $y=S q^{2^{s+1} k} z$ for some $z$, and $y \notin \mathrm{im} S q^{2}$ for $i \leqq s$.

Proof. $y$ projects nontrivially to $Q H^{*}\left(X ; \boldsymbol{Z}_{2}\right)$ because $\{y\} \in H^{*}\left(X ; \boldsymbol{Z}_{2}\right) / /$ $\xi H^{*}\left(X ; \boldsymbol{Z}_{2}\right)$ is odd degree primitive. Hence $\sigma^{*}(y)$ is nontrivial and is dual to an element $t \in H_{*}\left(\Omega X ; \boldsymbol{Z}_{2}\right)$ with $t^{2}=0$. Associated to the factorization

$$
S q^{v(s)+2^{s+2} k}=\sum_{u=-1} S q^{2^{s+2} k+2 v(s-1)-2 v(u)} Q_{u+1}
$$

is a secondary operation $\phi$. Now since $H_{*}\left(\Omega X ; \boldsymbol{Z}_{2}\right)$ is even dimensional, $Q_{u+1} \sigma^{*}(y)=0$ for all $u$. According to Zabrodsky [6], since $\Omega X$ is homotopy commutative,

$$
\bar{J} \phi\left(\sigma^{*} y\right) \in \sigma^{*} y \otimes \sigma^{*} y+\sum_{u=-1} \operatorname{im} S q^{2^{s+1} k+v(s-1)-v(u)} \otimes S q^{2^{s+1} k+v(s-1)-v(u)} .
$$

The proof now proceeds by induction. For $s=0$ the theorem follows because $Q^{4 k+1}=S q^{2 k} Q^{2 k+1}$. Assume the theorem is true for $s^{\prime}<s$. Then if degree $y=$ $v(s)+2^{s+2} k$. Suppose $y=S q^{2} z$ for some $i \leqq s$. Pick the smallest such $i$. Then $z^{2} \neq 0$ since $y^{2} \neq 0$. By induction $z=S q^{2^{i}+\cdots+2^{s-1+2}{ }^{s+1} k} w$ for some $u$. But then by applying Adem relations $S q^{2^{i}} z=y$ is in the image of $S q^{1}, \cdots, S q^{2^{i-1}}$. This contradicts the assumption that the smallest $i$ was chosen.

We conclude $y \notin \operatorname{im} S q^{2^{i}}$ for $i \leqq s$. It follows that $\sigma^{*} y \notin \operatorname{im} S q^{2^{i}}$ for $i \leqq s$, because, if so, $\sigma^{*} y=S q^{2^{i}} w$ and $w \in \bigotimes_{j} \wedge\left(\sigma^{*}\left(y_{j}\right)\right)$ which implies $w=\sigma^{*} z$.

Now by (3.1), if

$$
\sigma^{*} y \in \sum \operatorname{im} S q^{2 s+1 k+v(s-1)-v(u)}
$$

then $\left\langle t^{2}, \phi\left(\sigma^{*} y\right)\right\rangle \neq 0$ which would imply $t^{2} \neq 0$. This can't happen, so

$$
\sigma^{*} y \in \sum \operatorname{im} S q^{2^{s+1} k+v(s-1)-v(u)} .
$$


The only term not in the image of $S q^{2^{i}}$ for $i \leqq s$ is $S q^{2^{s+1} k}$. Hence $\sigma^{*} y=$ $S q^{2^{s+1} k} \sigma^{*} z$ which implies $y=S q^{2^{s+1 k}} z$.

Q.E.D.

\section{§4. Applications}

There are several applications of the fact that $Q^{1 k+1}=S q^{2 k} Q^{2 k+1}$. We describe a few which will be used in subsequent papers to deduce the action of the Steenrod algebra on $H^{*}(\Omega X)$.

Definition 4.1. Let $n$ have dyadic expansion

$$
n=2^{s_{1}}+2^{s_{2}}+\cdots+2^{s_{l}}
$$

where $0<s_{i}<s_{\imath+1}$. We will call $l$ the "length of $n$ ".

Proposition 4.1. If $4 k+1$ has length $l$ and $Q^{4 k+1}$ is nontrivial, then $Q^{2^{l}-1}$ is nontrivial.

Proof. From Theorem 2.3 and Theorem 2.1(a) we must have

$$
Q^{2 v(l)-1} \text { nontrivial. }
$$

By Theorem 2.2, $Q^{2^{l}-1}$ is nontrivial.

Q.E.D.

Proposition 4.2. $P H^{4 k}(\Omega X)=\xi\left(\sigma^{*} Q^{2 k+1}\right)$.

Proof. It is known that

$$
P H^{1 k}(\Omega X)=\sigma^{*} Q^{4 k+1} .
$$

But $Q^{4 k+1}=S q^{2 k} Q^{2 k+1}$.

Q.E.D.

Proposition 4.3. $Q^{s k+5}=S q^{2} Q^{s k+3}$

Proof. $Q^{8 k+j}=S q^{1 k+2} Q^{1 k+3}$

$$
=S q^{2} S q^{1 k} Q^{4 k+3}
$$

since $S q^{1 k+2}=S q^{2} S q^{4 k}$ modulo a $S q^{1}$ doubleton.

Q.E.D.

Definition 4.3. A coalgebra with squares is a coalgebra $C$ together with a map $\xi: C \rightarrow C$ of coalgebras such that degree $\xi x=$ twice degree of $x$.

Let $\xi: H^{*}\left(X ; \boldsymbol{Z}_{2}\right) \rightarrow H^{*}\left(X ; \boldsymbol{Z}_{2}\right)$ be the cup product squaring map. Define

$$
R=\left\{x \in H^{*}\left(X ; \boldsymbol{Z}_{2}\right) \mid \bar{\jmath} x \in \xi H^{*}\left(X ; \boldsymbol{Z}_{2}\right) \otimes H^{*}\left(X ; \boldsymbol{Z}_{2}\right)\right\} .
$$

Note that since $\xi H^{*}\left(X ; \boldsymbol{Z}_{2}\right)$ is a subHopf algebra, $\xi H^{*}\left(X ; \boldsymbol{Z}_{2}\right) \subseteq R$.

Further, by coassociativity,

$$
\bar{J} R \subseteq R \otimes R
$$

and in fact $R$ is a coalgebra over the Steenrod algebra with squares. 
Let $S(R)$ be the free commutative algebra on $R$. Then $S(R)$ is a Hopf algebra and there is a diagram

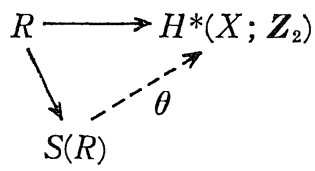

where the dotted arrow is a map of Hopf algebras.

Theorem 4.4. Let $I \subseteq S(R)$ be the ideal generated by elements of the form $x^{2}-\xi x$. Then $\theta$ induces an isomorphism $\tilde{\theta}$ of Hopf algebras over the Steenrod algebra

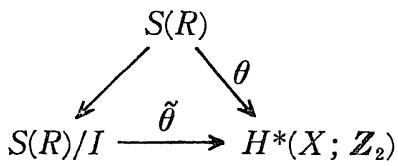

Proof. $\tilde{\theta}$ is a map of Hopf algebras. Because the map

$$
R \longrightarrow H^{*}\left(X ; \mathbb{Z}_{2}\right) \longrightarrow Q H^{*}\left(X ; \mathbb{Z}_{2}\right)
$$

is an epimorphism, $\tilde{\theta}$ is an epimorphism, with kernel $\xi R$. Filter both $S(R) / I$ and $H^{*}\left(X ; \boldsymbol{Z}_{2}\right)$ by powers of the augmentation ideal. Then $E_{0}(S(R) / I)$ is a primitively generated Hopf algebra on generators $R / \xi R$. Since generators of a Borel decomposition of $H^{*}\left(X ; \boldsymbol{Z}_{2}\right)$ can be chosen in $R, E_{0}\left(H^{*}\left(X ; \mathbb{Z}_{2}\right)\right)$ is also primitively generated on $R / \xi R$. It follows that $E_{0}(\tilde{\theta})$ is an isomorphism. Hence $\tilde{\theta}$ is an isomorphism.

Q.E.D.

\section{References}

[1] Browder, W., Torsion in $H$-spaces, Ann. of Math., 74 (1961), 24-51.

[2] Lin, J., Two torsion and the loop space conjecture, Ann. of Math., 115 (1982), $35-91$.

[3] Milnor, J. and Moore, J.C., On the structure of Hopf algebras, Ann. of Math., 81 (1965), 211-264.

[4] Thomas, E., Steenrod squares and $H$-spaces, I, II, Ann. of Math., 77 (1963), 306$317 ; 81$ (1965), 473-495.

[5] - Whitney Cartan product formulas, Math. Z. 118 (1970), 115-138.

[6] Zabrodsky, A., Cohomology operations and homotopy commutative $H$-spaces in the Steenrod algebra and its applications, Springer Verlag, 168 (1970), 308-317. 